Tropical Journal of Pharmaceutical Research May 2016; 15 (5): 1061-1068

ISSN: $1596-5996$ (print); 1596-9827 (electronic)

(C) Pharmacotherapy Group, Faculty of Pharmacy, University of Benin, Benin City, 300001 Nigeria.

All rights reserved.

Available online at http://www.tjpr.org

Original Research Article

http://dx.doi.org/10.4314/tjpr.v15i5.23

\title{
Patterns of prescribing and utilization of asthma medications in a tertiary hospital in Dubai, United Arab Emirates
}

\author{
Sahar A Fahmy ${ }^{1 *}$, Eman Abu-Gharbieh ${ }^{2}$ and Samer Hamidi ${ }^{3}$ \\ ${ }^{1}$ Department of Pharmaceutics, College of Pharmacy, Helwan University, ${ }^{2}$ Department of Pharmacology and Toxicology, Dubai \\ Pharmacy College, ${ }^{3}$ School of Health and Environmental Studies, Hamdan Bin Mohammad Smart University, Dubai, United \\ Arab Emirates \\ *For correspondence: Email: sahar33a@hotmail.com
}

Received: 7 October 2015

Revised accepted: 30 March 2016

\begin{abstract}
Purpose: To assess the prescribing patterns of asthma medications in a hospital in Dubai, United Arab Emirates (UAE) with regard to the demographic pattern of the population.

Methods: One hundred fifty four patients, 83 male and 71 female, were randomly selected from the outpatient respiratory diseases clinic of a tertiary hospital in Dubai, UAE over a 3-month period. Patients were asked to complete a structured questionnaire and data were analyzed using STATA 12 software.

Results: Most of the patients were within the age range of $0-10$ years. About $86 \%$ of the patients were overweight. Half of the patients were non-smokers while $51 \%$ of them had a family history of asthma. About $54 \%$ of the patients received multiple drug therapy of which two-drug combinations were widely prescribed (31\%). The most utilized drug classes were short acting $\beta$-agonists (42\%), xanthine drugs (16\%), leukotriene modifiers (14\%) and oral and intravenous corticosteroids (13\%). Statistical significant differences among the age groups $(F=2.33, p=0.0275)$ were found.

Conclusion: Primary prevention to reduce the level of exposure to common risk factors for asthma would be a vital step to control the disease. More resources should be channeled into educating physicians and patients on rational drug utilization to improve the quality of patients' care.
\end{abstract}

Keywords: Asthma, utilization of medicines, $\beta$-agonists, Xanthines, Leukotriene modifiers, Rational drug utilization

Tropical Journal of Pharmaceutical Research is indexed by Science Citation Index (SciSearch), Scopus, International Pharmaceutical Abstract, Chemical Abstracts, Embase, Index Copernicus, EBSCO, African Index Medicus, JournalSeek, Journal Citation Reports/Science Edition, Directory of Open Access Journals (DOAJ), African Journal Online, Bioline International, Open-J-Gate and Pharmacy Abstracts

\section{INTRODUCTION}

Drugs play an important role in improving human health and promoting well-being if used rationally. Drug utilization reviews (DUR) provide a powerful tool to assess knowledge and awareness of patients and physicians on the proper use of medications [1]. DUR is increasingly used as a tool for cost containment and quality assurance. There are three types of DUR reviews which are prospective, concurrent and retrospective. DURs help regulators implement the appropriate interventions to address certain issues in the health care systems. Among which is designing educational programs to improve prescribing practice and developing standard treatment protocols to treat specific diseases [2].

Asthma is a condition in which recurrent attacks of breathlessness and wheezing occurred mainly due to inflammation of the air passages. During 
asthma attack, airways are narrowed due to swelling of the lining of the passages causing reduction of the air flow [3]. In 2013 World Health Organization (WHO) estimated that 235 million people (3.5 percent of the world's population) currently suffer from asthma, and it is considered as the most common non-communicable disease among children [3]. The report on the global burden of asthma estimated that the number of disability-adjusted life years (DALYs) lost due to asthma was estimated to be around 15 million DALYs per year, and accounted for around one in every 250 deaths worldwide [4]. In UAE, asthma is considered one of the most predominant disease with about $8 \%$ to $10 \%$ of adults are suffering from asthma [5]. It was also reported that about $53 \%$ of children, and $17 \%$ of adults were missing school and work, respectively, due to asthma [6].

In another study conducted in Abu Dhabi, the capital of UAE, it was reported that around $10 \%$ of patients had made an emergency room visit because of Asthma. The direct cost of treating asthma in Abu Dhabi was estimated to be about 105 million AED a year, with $31 \%$ cost spent on medications only [7].

In the current study we investigated the pattern of drug use for asthma medications taking into consideration the demographic variations among patient population. This is done by conducting prescription audits and identifies irrationality in drug prescribing. Our main aim is to set recommendations for health regulators in order to take the appropriate measures to improve prescribing and hence improve quality of patient care.

\section{EXPERIMENTAL}

An observational study was planned to analyze the utilization patterns of anti-asthmatic drugs in a tertiary hospital in Dubai, UAE. The protocol of the study protocol was approved by hospital administration to conduct the study in the hospital. Two hundred patients visiting the outpatient respiratory diseases clinics were randomly selected during a period of three months (September to December 2012). Patients seen in the specialized clinics are followed up by respiratory diseases specialists or consultants. Data were collected through a structured questionnaire targeted asthmatic patients. The questionnaire was composed of two sections; the first set of questions were concerning patient demographic data i.e. age sex, occupation, level of education, family history and smoking status.
The second set of questions captured all relevant information related to the prescribed medications with dosing, frequency and route of administration.

\section{Inclusion criteria}

Patients diagnosed with asthma and are currently on anti-asthmatic medications were included in the study.

\section{Exclusion criteria}

Asthmatic patients who suffered from other diseases such as hypertension, diabetes, heart problems, bronchitis, chronic obstructive pulmonary disease (COPD), peptic ulcer, diabetes mellitus or any other co-morbidities were excluded.

Patients were randomly recruited in the study and they signed the informed consent form to indicate their participation in the study. They were interviewed and necessary information was collected as per the structured questionnaire. The prescriptions of participated patients diagnosed with asthma were collected and were interviewed for different parameters.

\section{Statistical analysis}

Descriptive statistics were carried out for all assessed parameters and results were expressed as frequencies and percentages. The data were analyzed for different parameters using One-way analysis of variance (ANOVA). $P$ $<0.05$ was taken to denote statistical significance. All statistical analysis was conducted using STATA (StataCorp, College Station, Texas, US) version 12.

\section{RESULTS}

\section{Demographic characteristics}

Among the 200 randomly selected patients, only 154 patients were eligible to be included in the study as per the inclusion criteria. Around $54 \%$ of the study population was males with most of the patients diagnosed with asthma were within the young age group (0-10) years. About $86 \%$ of the patients were overweight. Most of the patients are well educated with $54.6 \%$ of patients have graduate degree. About $23 \%$ of patients were UAE nationals and about $77 \%$ were expatriates. Patient smoking history was assessed and determined to be of equal distribution among patients with $51 \%$ of patients were none smokers. About $51.3 \%$ of patients 


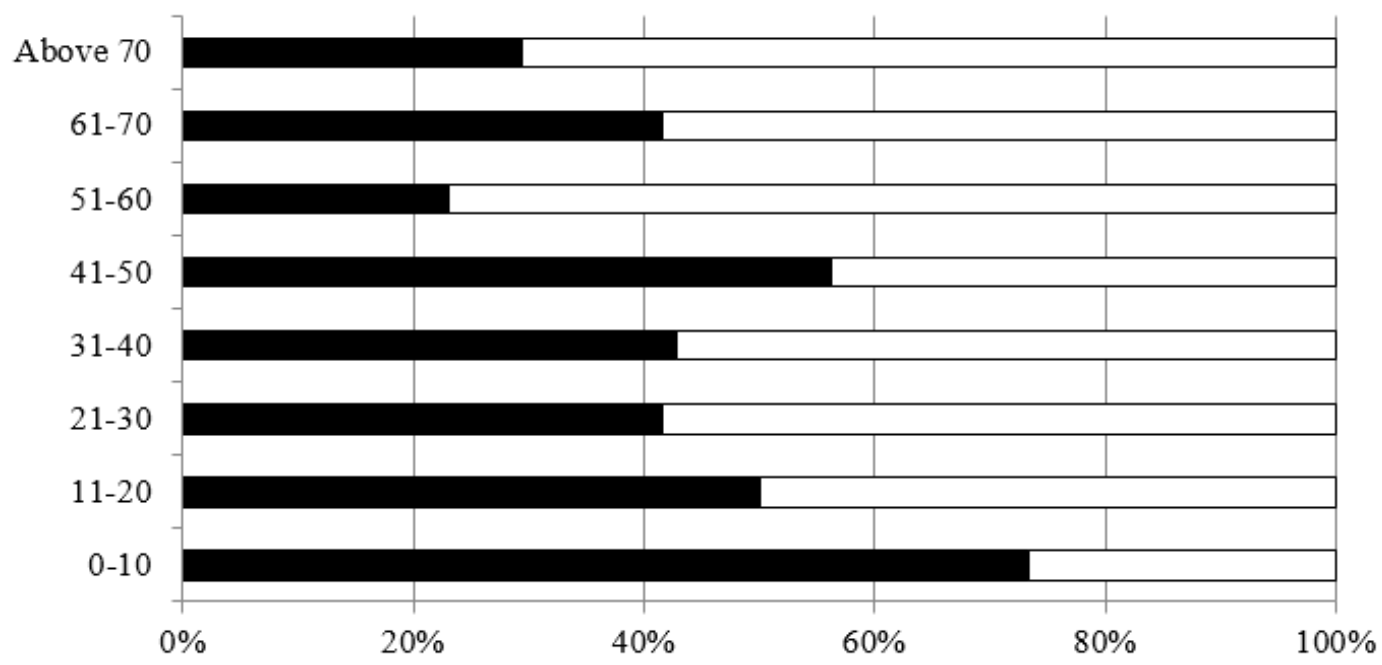

Figure 1: Age and gender distribution of study participants diagnosed with asthma

Table 1: Patients' demographic data

\begin{tabular}{ll}
\hline Demographic information & Number (\%) \\
\hline Age (years) & $64(41.6)$ \\
$0-10$ & $6(3.9)$ \\
$11-20$ & $12(7.8)$ \\
$21-30$ & $14(9.1)$ \\
$31-40$ & $16(10.4)$ \\
$41-50$ & $13(8.4)$ \\
$51-60$ & $12(7.8)$ \\
$61-07$ & $17(11.0)$ \\
770 & \\
Gender & $83(53.9)$ \\
Male & $71(46.1)$ \\
Female & \\
Body mass index & $5(3.2)$ \\
Underweight & $17(11.1)$ \\
Normal & $132(85.7)$ \\
Overweight & \\
Level of education & $70(45.5)$ \\
<High school & $3(1.9)$ \\
High school & $81(52.6)$ \\
Graduate degree & \\
Nationality & $36(23.3)$ \\
UAE national & $118(76.6)$ \\
Expatriate & \\
Socioeconomic status & $5(3.2)$ \\
Low & $30(19.4)$ \\
Lower middle & $118(76.6)$ \\
Upper middle & $1(0.4)$ \\
High & \\
Occupation & $19(12.3)$ \\
Employed worker & $42(27.3)$ \\
Employed non-worker & $60(39.0)$ \\
Not employed & $33(4.5)$ \\
Retired & $80(51.9)$ \\
Smoking History & $49(31.8)$ \\
Never smoke & $25(16.3)$ \\
Current smoker & \\
Ex-smoker & \\
Family History of Asthma & \\
Yes & \\
No & $(28.6)$ \\
Do not know & \\
\hline
\end{tabular}

had a family history of asthma. The majority of patients participated in the study $(76.6 \%)$ were with upper middle socioeconomic status. Table 1 summarizes patients' demographic data.

\section{Age-gender distribution of patients diagnosed with asthma}

To capture the age gender distribution among participants, gender data was analyzed for the eight age groups. It was found that male group were highly distributed within two age groups with 46 (72\%) and 9 (56\%) study participants aged between (0-10) years and (40-50) years respectively. However, the female group was highly distributed within two age group with 10 $(77 \%)$ and12 $(71 \%)$ of study participants aged between (51-60) years and (above 70) years respectively. Figure 1, shows age and gender distribution of patients with asthma. A statistical analysis was done for age- gender distribution using (ANOVA) and the results showed a significant difference of gender distribution among the different age groups with a $p$-value of 0.0275

A total of 83 (54\%) of study participants received multiple drug therapy compared to 71 (46\%) of participants who received single drug therapy. In multiple drug therapy, two-drug combinations were widely prescribed 48 (31 \%) than combinations of three 24 (16\%) or four drugs 11 (7\%) as shown in Table 2.

The pattern of drug prescription for treating asthma at the study showed that short acting $\beta$ agonist (SABA) are the highest prescribed class of medications (42\%) followed by xanthines (16 
$\%)$,leukotriene modifiers (14 \%) and corticosteroids (13\%) as shown in Table 3.

Table 2: Multiple versus single drug therapy

\begin{tabular}{lc}
\hline $\begin{array}{l}\text { Number } \\
\text { of drugs }\end{array}$ & Number of patients (\%) \\
\hline 1 & $92(46 \%)$ \\
2 & $63(31 \%)$ \\
3 & $33(16 \%)$ \\
4 & $15(7 \%)$ \\
\hline
\end{tabular}

Table 3: Types of drugs used to treat asthma

\begin{tabular}{lc}
\hline Drug type & $\begin{array}{c}\text { Drug } \\
\text { utilization }\end{array}$ \\
\hline Short acting beta agonist (SABA) & $\mathbf{4 2} \%$ \\
Xanthines & $\mathbf{1 6} \%$ \\
Leukotriene Modifiers & $\mathbf{1 4} \%$ \\
Oral and IV corticosteroids & $\mathbf{1 3} \%$ \\
Inhaled corticosteroids (ICS) & $9 \%$ \\
Antibiotics & $3 \%$ \\
Long acting beta agonist (LABA) & $\mathbf{2} \%$ \\
Combination Inhalers & $\mathbf{1} \%$ \\
Grand Total & $100 \%$ \\
\hline
\end{tabular}

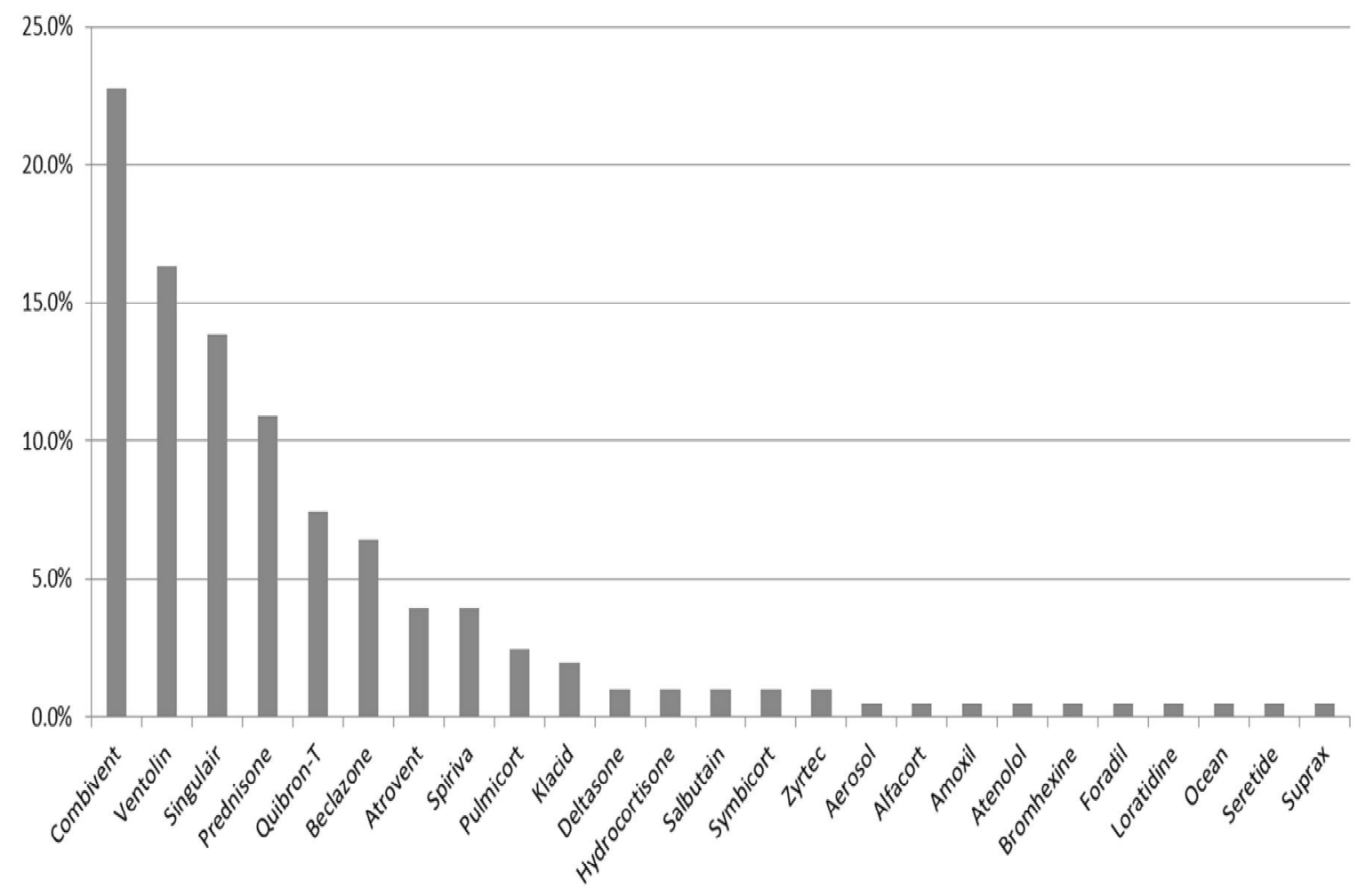

Figure 2: Most commonly used drugs for the treatment of asthma

The most commonly prescribed drug was Combivent ${ }^{\circledR}$ which is composed of salbutamol and ipratropium bromide with a ratio of $23 \%$ followed by Ventolin® (salbutamol, $16 \%$ ) and Singular ${ }^{\circledR}$ (montelukast, $14 \%$ ) as shown in Figure 2. Salbutamol or albuterol were chosen as the first line drug in the management of asthma and were used as required by patients for the symptomatic relief of breathlessness and wheezing.

The use of all asthma medication classes was determined to be significantly different among the different age group $(\mathrm{F}=2.33, p=0.0275)$. The 0 -10 years age group showed the highest rate of drug utilization (32\%).
Drug utilization was assessed based on gender distribution and found to around (47\%) for males versus $(53 \%)$ for females. There was no statistical significant difference among male and female patients in terms of utilizations of asthma medications $(\mathrm{F}=2.55, p=0.1124)$.

\section{DISCUSSION}

Drug therapy is used to relieve and prevent symptoms of asthma and treat exacerbations. Results of the study showed that drug classes commonly used to treat asthma include shortacting beta agonists which are used for shortterm symptom relief; xanthine drugs, leukotriene modifiers, oral and intravenous corticosteroids which are used during episodes of more severe asthma; and inhaled corticosteroids and long- 
acting beta agonists, used for symptom prevention and disease control. Over the years, inhaled corticosteroids have dominated asthma treatment [8]. However, medical reports showed that the more the asthma patients used inhaled steroids, the weaker their bones became, thus increasing the risk of osteoporosis [9].

Most people who are on inhaled corticosteroids use them intermittently, even though guidelines recommend that these drugs be used regularly by those who need them [10]. Most adults also use the strongest formulations of inhaled corticosteroids, even though less potent formulations are often effective and have fewer side effects [10]. The results showed that about only $9 \%$ of patients use inhaled corticosteroids, which is a good sign of rational drug utilization.

Another therapeutic category used for the management of asthma is leukotriene modifiers with $14 \%$ of study participants on the treatment alone or in combination with other medications. The ability of leukotriene receptor antagonistbased medication, to be used along with inhaled beta-agonists or added to existing asthma treatment regimens; allow reduction of a concomitant therapy. Innovative drugs are the leukotriene receptor antagonists which are playing an important role in asthma therapy and offer advantages over other currently available treatments, including excellent tolerability, oral route of administration, once-daily dosage and proven efficacy in a wide range of patients, including children.

The use of short acting drug relievers alone was evidenced not to control asthma properly. People with asthma who rely on short-acting beta agonists, without using inhaled corticosteroids, have been shown to be more likely to suffer exacerbations or attacks of asthma requiring an emergency department visit or an admission to hospital [11]. In our study most of study participants received salbutamol. Salbutamol is the preferred Short acting beta-2 agonist because it has an excellent safety profile. Rapid reversal of airflow obstruction is best achieved by repetitive administration of salbutamol nebulisation which is considered to be the first medication of choice and it is lifesaving.

Utilization of asthma medication was highest in early childhood from the age range of (0-10 years) and late in elderly (over 70 years). This might be due the fact that elderly are especially prone to the adverse health effects of chronic obstructive pulmonary disease (COPD), which is a common disorder in that population [12].

\section{Limitations of the study}

The study was conducted in one hospital only. Expanding the study to different settings is needed to assess prescribing practices in those settings. Assessment of illness severity was not possible due to lack of access to patient medical records. Thus, patients could not be classified into different groups based on severity and could not evaluate whether the prescribing patterns were in line with international standards. In addition, the effect of data based on only one visit to a patient in outpatient settings on the assessment of how prescribing patterns changed over the course of illness in patients remains unclear.

\section{CONCLUSION}

Asthma creates substantial burden to individuals and families and often restricts individuals' activities for a lifetime.

The present prescribing practice in the management of asthma in the setting of this study is not sufficiently rational. The presented data should be considered a baseline for the current practice and may be used in proposing appropriate interventions to improve current prescribing trend for a more rational utilization of asthma medications. It is also recommended that to develop asthma treatment guidelines and monitor physician adherence to such guidelines in order to manage asthmatic patients effectively. More steps are required to implement regulatory and managerial interventions needed to promote rational prescribing of asthma medications.

\section{ACKNOWLEDGEMENT}

The authors would like to thank Rama Al-Kateb, Aya Kayali and Aiea Mohammad who helped with the data collection phase of this study.

\section{CONFLICT OF INTEREST}

No conflict of interest associated with this work.

\section{CONTRIBUTION OF AUTHORS}

We declare that this work was done by the authors named in this article and all liabilities pertaining to claims relating to the content of this article will be borne by the authors. 


\section{REFERENCES}

1. Carruthers AA, Krska J. Thrombolytics. A drug utilization review in a district general hospital. J Clin Pharm Ther 1997; 22: 335-338.

2. Hammerman A, Greenberg A, Yinnon AM. Drug use evaluation of ciprofloxacin: impact of educational efforts on appropriateness of use. J Clin Pharm Ther 1997; 22. 415-420.

3. WHO Chronic respiratory diseases: Asthma definition. World Health Organization [cited 2014 Nov 20]. Available from: http://www.who.int/respiratory/asthma/definition/en

4. Masoli M, Fabian D, Holt S, Beasley R. The global burden of asthma: executive summary of the GINA Dissemination Committee report. Allergy 2004; 59: 469478.

5. Hassan MBH, Santhakumar S, Soriano JB, Pawankar $R$. Asthma insights and reality in the United Arab Emirates. Ann Thorac Med 2010; 5: 217-221.

6. Mahboub BH, Al-Hammadi S, Rafique M, Sulaiman N, Pawankar R, Al Redha Al, Mehta AC. Population prevalence of asthma and its determinants based on European Community Respiratory Health Survey in the United Arab Emirates. BMC Pulm Med 2012; 12: 4.
7. Alzaabi A, Alseiari M, Mahboub B. Economic burden of asthma in Abu Dhabi: A retrospective study. Clinicoecon Outcomes Res 2014; 6: 445-450.

8. WebMd Asthma Health centre. Inhaled corticosteroids for long-term control of asthma. Healthwise, Inc; 1995-2015 [cited 2 March 2015]. Available from: http://www.webmd.com/asthma/inhaled-corticosteroid sfor-long-term-control-of-asthma

9. Web MD Asthma Health centre. Steroid inhalers for asthma can weaken bones. Healthwise, Inc; 1995-2015 [cited 2 March 2015]. Available from: http://www. webmd.com/asthma/news/20000421/asthma-steroidinhalers-bone-loss

10. Australian Centre for Asthma Monitoring. Patterns of asthma medication use in Australia. Cat. no. ACM 11. Canberra: AlHW. 2007 [cited 4 April 2014]. Available from:http://www.aihw.gov.au/WorkArea/DownloadAsset. aspx?id=6442453775

11. Anis $A H$, Lynd $L D$, Wang $X H$, King G, Spinelli JJ, Fitzgerald M, Bai T, Pare P. Double trouble: impact of inappropriate use of asthma medication on the use of health care resources. CMAJ 2001; 164: 625-631.

12. Nicola A. Hanania, Gulshan Sharma, and Amir Sharafkhaneh. COPD in the Elderly Patient. Semin Respir Crit Care Med. 2010; 31(5): 596-606. Available from: http://www.medscape.com/viewarticle/730813 